\title{
ON CONJUGACY SEPARABILITY OF FUNDAMENTAL GROUPS OF GRAPHS OF GROUPS
}

\author{
M. SHIRVANI
}

\begin{abstract}
A complete determination of when the elements of a fundamental group of a (countable) graph of profinite groups are conjugacy distinguished is given. By embedding an arbitrary fundamental group $G$ into one with profinite vertex groups and making use of the above result, questions on conjugacy separability of $G$ can be reduced to the solution of equations in the vertex groups of $G$.
\end{abstract}

\section{INTRODUCTION}

An element $g$ of a group $G$ is said to be conjugacy distinguished (or conjugacy separable) in $G$ if for every element $h$ of $G$ not conjugate to $g$ there exists $N \triangleleft_{f} G$ such that $g N$ and $h N$ are nonconjugate in $G / N$. A group is conjugacy separable if all its elements are conjugacy distinguished. The bestknown classes of conjugacy separable groups are polycyclic-by-finite groups [10], profinite groups [11], free-by-finite groups [5], and certain Fuchsian groups [19]. Results are also known on conjugacy separability of certain amalgamated free products (e.g., of free groups [18]), and some one-relator groups with torsion [1]. In the last mentioned paper the authors ask whether every one-relator group with torsion is conjugacy separable. More generally, one can ask when the fundamental group of a graph of groups is conjugacy separable.

In order to investigate these problems one might adopt the following strategy: let $G$ be a residually finite group, with $\widehat{G}$ its profinite completion (i.e., $\widehat{G}$ is the inverse limit of the system of finite images $G / N$ of $G$ ). It is easily shown that if $g, h \in G$ are conjugate in every finite image of $G$, then they are conjugate in $\widehat{G}$, and so $G$ is conjugacy separable if and only if whenever $g, h \in G$ are conjugate in $\widehat{G}$, then they are conjugate in $G$. Unfortunately, if $G$ is a nontrivial fundamental group of a graph of groups, then $\widehat{G}$ is still not sufficiently well understood (cf. [7, 20]) to allow for the successful completion of the last step. A complete answer can be given when the vertex groups are themselves profinite groups. This, in turn, allows necessary and sufficient conditions to be derived for the general case.

Let $X$ be a connected graph, let $\mathscr{G}=\left\{A_{x}, x \in V X, H_{e}, e \in E X\right\}$ be a graph of groups over $X$, and let $G=\pi_{1}(\mathscr{G}, X)$ be the fundamental group of

Received by the editors September 21, 1990.

1980 Mathematics Subject Classification (1985 Revision). Primary 20E26; Secondary 20E06, $20 \mathrm{E} 18$. 
this graph of groups (see [2, 4], or [14] for the relevant definitions and properties). We assume that $G$ is residually finite, and denote by $\widehat{A}_{x}$ the profinite completion of the vertex group $A_{x}$ with respect to the induced topology $\left\{A_{x} \cap M: M \triangleleft_{f} G\right\}$. Let $\bar{H}_{e}$ denote the topological closure of $H_{e}$ in $\hat{A}_{i(e)}$. The edge isomorphisms can be extended to these closures (cf. Lemma 3.1). The result is a graph of groups over $X$ with fundamental group $G^{+}$(for details see §3). The obvious maps $A_{x} \rightarrow \widehat{A_{x}}$ extend to an embedding of $G$ into $G^{+}$. If we denote the conjugacy class of $g$ in $G$ by $g^{G}$ and $\bigcap_{M \triangleleft_{f} G} g^{G} M$ by $\operatorname{cl}_{G}\left(g^{G}\right)$, then we have:

Theorem 3.4. For every $g \in G$ we have $\operatorname{cl}_{G}\left(g^{G}\right)=\operatorname{cl}_{G^{+}}\left(g^{G^{+}}\right) \cap G$.

The point of this result is that most elements of $G^{+}$turn out to be conjugacy distinguished (i.e, $\operatorname{cl}_{G^{+}}\left(g^{G^{+}}\right)=g^{G^{+}}$). If $g \in G$ is such an element, then $g$ is conjugacy distinguished in $G$ if and only if $g^{G^{+}} \cap G=g^{G}$. This condition is quite tractable, and amounts to whether certain equations hold in the vertex groups $A_{x}$ and $\widehat{A}_{x}$. Assuming that we have enough information about the vertex groups and their completions, the conjugacy separability of $G$ can be decided.

To state the results for $G^{+}$, let $Y$ be a maximal subtree of $X$, and write $A^{+}=\left\langle\hat{A}_{x}: x \in V X\right\rangle \leq G^{+}$. Then $A^{+}$is the fundamental group of the graph of groups $\mathscr{G}$ restricted to $Y$, and $G^{+}$is an HNN-extension with base group $A^{+}$. As a consequence of $4.4,4.6$, and 4.9 below we get

Theorem A. Let $a \in A^{+}$.

(i) If either no conjugate of a belongs to a vertex group, or $X$ is locally finite, $a \in \hat{A}_{x}$, and no $\hat{A}_{x}$-conjugate of a belongs to an edge subgroup, then $a$ is conjugacy distinguished in $A^{+}$.

(ii) Assume that $X$ is locally finite, let $D=\bigcup_{e \in E X} \bar{H}_{e}$, and suppose a $\in$ $D$ belongs to only a finite number of edge subgroups. Then $a$ is conjugacy distinguished in $A^{+}$if and only if there exist vertices $x_{1}, \ldots, x_{m}$ of $Y$ such that $D \cap a^{A^{+}}=D \cap\left\{a^{a_{1} \cdots a_{m}}: a_{i} \in \widehat{A_{x_{i}}}\right\}$.

For elements of $G^{+}$we obtain from 5.1, 5.3, 5.6, and 5.8

Theorem B. (i) All elements of $G^{+} \backslash A^{+}$, and all elements of $A^{+}$satisfying condition (i) of Theorem A, are conjugacy distinguished in $G^{+}$.

(ii) Assume that $X$ is locally finite, and let $D^{\prime}=\bigcup_{e \in E X \backslash E Y} \bar{H}_{e}$ and $C=$ $\bigcup_{x \in V X} \widehat{A_{x}}$.

Then $h \in D^{\prime}$ is conjugacy distinguished in $G^{+}$if and only if $h^{G^{+}} \cap C$ is a union of a finite number of sets of the form $C \cap\left\{h^{g}: g \in \widehat{A}_{x_{1}} t_{e_{1}}^{\varepsilon_{1}} \cdots \hat{A}_{x_{n}} t_{e_{n}}^{\varepsilon_{n}} \widehat{A}_{x_{n+1}}\right.$, for some $e_{i} \in E X \backslash E Y, \varepsilon_{i}= \pm 1$, and $\left.x_{i} \in V X\right\}$.

This in particular settles the problem for the case when the vertex groups are profinite, since such a $G$ can be residually finite if and only if $G \cong G^{+}$, by 3.3 below.

\section{NotATION}

Let $X$ be a directed connected graph, $\mathscr{G}$ a graph of groups $\left\{A_{x}: x \in\right.$ $V X ; H_{e}: e \in E X$ with isomorphisms $\theta_{e}$ from $H_{e} \leq A_{i(e)}$ to $\left.H_{\bar{e}} \leq A_{t(e)}\right\}$ over 
$X$, and $G=\pi_{1}(\mathscr{G}, X)$ the fundamental group of $(\mathscr{G}, X)$ relative to the choice of a fixed maximal subtree $Y$ (see [2, 4], or [14] for details). It is well known that if $A=\pi_{1}\left(\left.\mathscr{G}\right|_{Y}, Y\right)$ then the obvious map of $A$ to $\left\langle A_{x}: x \in V Y\right\rangle \subseteq G$ is an isomorphism, and $G$ is an HNN-extension with base group $A$ and stable letters $t_{e}$ corresponding to the edges $e \in E X \backslash E Y$ :

$$
G=\left\langle A, t_{e}: t_{e}^{-1} h t_{e}=h \theta_{e} \text { for all } h \in H_{e}, e \in E X \backslash E Y\right\rangle .
$$

In the notation of [16] let $I=I(\mathscr{G}, X)$ denote the set of all sequences $\left(P_{x}\right)_{x \in V X}$ satisfying the following conditions: (a) $P_{x} \triangleleft A_{x}$ and there exists an integer $m=m(P)$ such that $\left|A_{x}: P_{x}\right| \leq m$ for all $x \in V X$, (b) $\left(P_{i(e)} \cap H_{e}\right) \theta_{e}=P_{t(e)} \cap H_{\bar{e}}$ for all $e \in E X$. For $P, Q \in I$ we write $P \leq Q$ if $P_{x} \subseteq Q_{x}$ for all $x$. For $P \in I$ let $\mathscr{G}_{P}$ denote the graph of groups $\left\{A_{x} / P_{x}, H_{e} P_{i(e)} / P_{i(e)}\right.$, induced isomorphisms $\left.\theta_{e, P}\right\}$, and let $G_{P}=\pi_{1}\left(\mathscr{G}_{P}, X\right)$. The projections $A_{x} \rightarrow A_{x} / P_{x}$ clearly extend to an epimorphism $\pi_{P}: G \rightarrow G_{P}$. It is well known that $G_{P}$ is free-by-finite (e.g., [14, Exercise 2 on p. 123]). Also note that if $M \triangleleft_{f} G$ then $P=\left(M \cap A_{x}\right)_{x \in V X} \in I$, and $G / M$ is a homomorphic image of $G_{P}$. In particular, this implies that if $G$ is residually finite then $\bigcap_{P \in I} P_{x}=\langle 1\rangle$ for all $x \in V X$. We assume this from now on. For $g \in G$ write $g^{G}$ for the conjugacy class of $g$ in $G$.

Proposition 2.1. Let $g \in G$. Then $\bigcap_{M_{\triangleleft_{f}} G} g^{G} M=\bigcap_{P \in I} g^{G} \operatorname{ker} \pi_{P}$. In particular, $\bigcap_{M \triangleleft_{f} G} M=\bigcap_{P \in I} \operatorname{ker} \pi_{P}$.

Proof. If $M \triangleleft_{f} G$ induces $P \in I$ then $M \supseteq \operatorname{ker} \pi_{P}$, so $g^{G} M \supseteq \bigcap_{P} g^{G} \operatorname{ker} \pi_{P}$. Conversely, given $P \in I$ let $P \uparrow=\left\{M \triangleleft_{f} G: M \cap A_{x}=P_{x}\right.$ for all $\left.x \in V X\right\}$. Now by a theorem of Dyer [5], $G_{P}$ is conjugacy separable, which implies that

$$
\left(g \pi_{P}\right)^{G_{P}}=\bigcap_{M \in P \uparrow}\left(g \pi_{P}\right)^{G_{P}} M \pi_{P}=\bigcap_{M \in P \uparrow}\left(g^{G} M\right) \pi_{P},
$$

whence $g^{G} \operatorname{ker} \pi_{P}=\bigcap_{M \in P \uparrow} g^{G} M$. The result follows. (For the final part take $g=1$.

\section{Profinite closures}

It is evident that if $P, Q \in I$, then $P \cap Q=\left(P_{x} \cap Q_{x}\right)_{x \in V X}$ also belongs to $I$. We refer to the topology on $A_{x}$ with $\left\{P_{x}: P \in I\right\}$ as a fundamental system of open neighbourhoods of the identity as the $I$-topology. For each $x$ let $\widehat{A_{x}}=\lim _{P \in I}\left(A_{x} / P_{x}\right)$, the inverse limit being formed relative to the partial order $\leq$ of $I$ introduced above. Let $\bar{H}_{e}$ denote the topological closure of $H_{e}$ in $\widehat{A}_{i(e)}$. We have

Lemma 3.1. For every $e \in E X$, the isomorphism $\theta_{e}: H_{e} \rightarrow H_{\bar{e}}$ extends to an isomorphism $\bar{\theta}_{e}: \bar{H}_{\varepsilon} \rightarrow \bar{H}_{\bar{e}}$ such that $\left(\bar{H}_{e} \cap \bar{P}_{i(e)}\right) \bar{\theta}_{e}=\bar{H}_{\bar{e}} \cap \bar{P}_{t(e)}$.

Proof. For each $P \in I$ there are canonical isomorphisms

$$
\begin{aligned}
\bar{H}_{e} /\left(\bar{H}_{e} \cap \bar{P}_{i(e)}\right) & \cong \bar{H}_{e} \bar{P}_{i(e)} / \bar{P}_{i(e)}=H_{e} \bar{P}_{i(e)} / \bar{P}_{i(e)} \\
& \cong H_{e} /\left(H_{e} \cap \bar{P}_{i(e)}\right)=H_{e} / H_{e} \cap P_{i(e)},
\end{aligned}
$$

and $\bar{H}_{e}$ is naturally isomorphic to the inverse limit $\lim _{P \in I}\left(H_{e} / H_{e} \cap P_{i(e)}\right)$ [12].

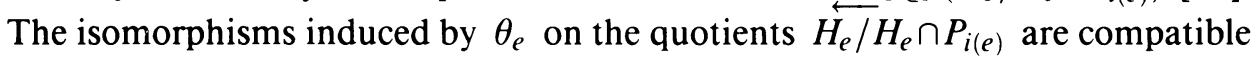


with the inverse limit structure, so the existence of $\bar{\theta}_{e}$ follows. (If one thinks of the inverse limit $\widehat{A_{x}}$ as a subgroup of the cartesian product $\prod_{x \in V X}\left(A_{x} / P_{x}\right)$, then the image of the element $h=\left(h_{P} \pi_{P}\right) \in \bar{H}_{e}$ is $h \bar{\theta}_{e}=\left(h_{P} \theta_{e} \pi_{P}\right) \in \bar{H}_{\bar{e}}$.) The intersection property of $\bar{\theta}_{e}$ is also trivial from the construction.

Thus we have a graph of groups $\mathscr{G}^{+}=\left\{\hat{A}_{x}, \bar{H}_{e}, \bar{\theta}_{e}\right\}$ over $X$. Let $G^{+}=$ $\pi_{1}\left(\mathscr{G}^{+}, X\right)$. The natural embeddings $A_{x} \rightarrow \hat{A}_{x}$ extend to a homomorphism $\mu: G \rightarrow G^{+}$. The next few results exploit the relationship between $G^{+}$and the residual properties of $G$.

Lemma 3.2. Let $G, G^{+}$, and $\mu$ be as above. Then $G^{+}$is residually finite and $\operatorname{ker} \mu=\bigcap_{M \triangleleft_{f} G} M$.

Proof. The edge subgroups of $G^{+}$are closed in their vertex groups by definition, and 3.1 implies that if $P \in I(\mathscr{G})$ then $\bar{P}=\left(\bar{P}_{x}\right) \in I\left(\mathscr{G}^{+}\right)$. The residual finiteness of $G^{+}$is therefore a consequence of the theorem of [16]. For the second part let $P \in I$, and consider the following diagram with exact top row:

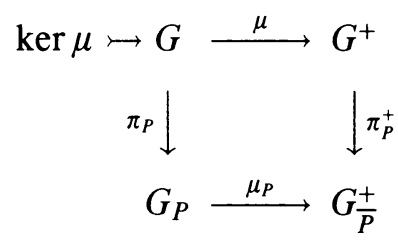

The diagram is easily seen to commute, and $A_{x} / P_{x} \cong \hat{A}_{x} / \bar{P}_{x}$ implies that $\mu_{P}$ is an isomorphism. Then $1=(\operatorname{ker} \mu) \mu \pi_{P}^{+}=(\operatorname{ker} \mu) \pi_{p} \mu_{P}$ implies that $\operatorname{ker} \mu \subseteq$ $\operatorname{ker} \pi_{P}$ for all $P$, so $\operatorname{ker} \mu \subseteq \bigcap_{P} \operatorname{ker} \pi_{P}=\bigcap_{M_{\triangleleft} G} M$ by 2.1 . On the other hand, $G / \operatorname{ker} \mu$ is a subgroup of $G^{+}$; being residually finite, the reverse inclusion follows.

Before proceeding further we mention that, in general, profinite closedness of the edge subgroups in their vertex groups is not necessary for the residual finiteness of $G$ (cf. $[15,17])$. In the case of profinite vertex groups, however, we have the following result.

Corollary 3.3. Let $G=\pi_{1}(\mathscr{G}, X)$, and assume that $A_{x}=\lim _{P \in I}\left(A_{x} / P_{x}\right)$ for all $x \in V X$. Then $G$ is residually finite if and only if every edge subgroup is profinitely closed in its vertex group.

Proof. Suppose there exists an element $a \in \bar{H}_{e} \backslash H_{e}$ for some $e \in E X$. If $e$ is an edge in the maximal subtree $Y$ let $g=a^{-1}\left(a \bar{\theta}_{e}\right)$; otherwise let $g=$ $a^{-1} t_{e}\left(a \bar{\theta}_{e}\right) t_{e}$. Then $g \neq 1$ in $G$, while $g \mu=1$, so $G$ is not residually finite by 3.2. The converse follows from 3.2 and the fact that when the edge subgroups are closed we have $G=G^{+}$.

If $S$ is a subset of a group $H$, write $\operatorname{cl}_{H}(S)$ for $\bigcap_{M \triangleleft_{f} H} S M$.

Theorem 3.4. Let $G, G^{+}$, and $\mu$ be as above. If $g \in G$ then $\operatorname{cl}_{G}\left(g^{G}\right) \mu=$ $\mathrm{cl}_{G^{+}}\left((g \mu)^{G^{+}}\right) \cap G \mu$.

We are not assuming that $\mu$ is injective. 
Proof. Let $S=\operatorname{cl}_{G}\left(g^{G}\right)=\bigcap_{P \in I} g^{G} \operatorname{ker} \pi_{P}$ (by 2.1). Then using $\operatorname{ker} \mu \subseteq \operatorname{ker} \pi_{P}$ (cf. the proof of 3.2) we have

$$
S \mu=\bigcap_{P \in I}\left(g^{G} \operatorname{ker} \pi_{P}\right) \mu=\bigcap_{P \in I}(g \mu)^{G \mu}\left(\operatorname{ker} \pi_{P}\right) \mu .
$$

Now it is easy to see from the commutative diagram in the proof of 3.2 and the fact that $\mu_{P}$ is an isomorphism, that $\left(\operatorname{ker} \pi_{P}\right) \mu=\operatorname{ker} \pi_{P}^{+} \cap G \mu$. The proof of 2.1 applies without change to show that $\operatorname{cl}_{G^{+}}\left(y^{G^{+}}\right)=\bigcap_{P \in I} y^{G^{+}} \operatorname{ker} \pi_{P}^{+}$for all $y \in G^{+}$. Thus

$$
S \mu=\bigcap_{P \in I}(g \mu)^{G \mu}\left(\operatorname{ker} \pi_{P}^{+} \cap G \mu\right)=\bigcap_{P \in I}(g \mu)^{G \mu} \operatorname{ker} \pi_{P}^{+} \cap G \mu,
$$

which is certainly contained in $\bigcap_{P \in I}(g \mu)^{G^{+}} \operatorname{ker} \pi_{P}^{+} \cap G \mu$. Conversely, suppose we have an element $z \mu \in \bigcap_{P \in I}(g \mu)^{G^{+}} \operatorname{ker} \pi_{P}^{+}$, where $z \in G$. Then for each $P$ there exists $w_{P} \in G^{+}$such that $z \mu \pi_{P}^{+}=\left(w_{P}^{-1}(g \mu) w_{P}\right) \pi_{P}^{+}$. Since $\mu_{P}$ is an isomorphism, there exists $u_{P} \in G$ such that $u_{P} \pi_{P} \mu_{P}=w_{P} \pi_{P}^{+}$. The above equation now becomes $z \pi_{P} \mu_{P}=\left(u_{P}^{-1} g u_{P}\right) \pi_{P} \mu_{P}$, and since $\mu_{P}$ is injective we obtain $z \in \bigcap_{P \in I} g^{G} \operatorname{ker} \pi_{P}=S$. We have now shown that

$$
S \mu=\bigcap_{P \in I}(g \mu)^{G \mu} \operatorname{ker} \pi_{P}^{+} \cap G \mu \subseteq \bigcap_{P \in I}(g \mu)^{G^{+}} \operatorname{ker} \pi_{P}^{+} \cap G \mu \subseteq S \mu,
$$

as required.

Since conjugacy separable groups are residually finite, we henceforth assume that $\operatorname{ker} \mu=\langle 1\rangle$, and identify $G$ with $G \mu$. The statement of 3.4 can then be written more simply as $\operatorname{cl}_{G}\left(g^{G}\right)=\operatorname{cl}_{G^{+}}\left(g^{G^{+}}\right) \cap G$. What is the point of this result? Let $g$ and $g^{\prime}$ be elements of $G$ which are conjugate in every finite image of $G$. By 2.1 this means that for every $P \in I$ there exists $w_{P} \in G$ such that $g^{\prime} \pi_{P}=\left(w_{P}^{-1} g w_{P}\right) \pi_{P}$, and at first glance it is conceivable that the "length" of the elements $w_{P}$ (in whatever sense) might be unbounded. Now if we have $\operatorname{cl}_{G^{+}}\left(g^{G^{+}}\right)=g^{G^{+}}$, then 3.4 implies that $g^{\prime} \in \operatorname{cl}_{G}\left(g^{G}\right)=g^{G^{+}} \cap G$, so $g^{\prime}=w^{-1} g w$, where $w \in G^{+}$. This, of course, means that the above $w_{P}$ can be chosen to have bounded length. It turns out that conjugacy separability is commonplace for elements of $G^{+}$. Before we proceed with this, we state the following, which does not require $\operatorname{ker} \mu=\langle 1\rangle$.

Corollary 3.5. If $g$ is conjugacy distinguished in $G$, then

$$
(g \mu)^{G^{+}} \cap G \mu=g^{G} \mu .
$$

Proof. For if $(g \mu)^{G^{+}} \cap G \mu=S \mu$, where $S \neq g^{G}$, then

$$
g^{G} \mu=\operatorname{cl}_{G}\left(g^{G}\right) \mu=\operatorname{cl}_{G^{+}}\left((g \mu)^{G^{+}}\right) \cap G \mu \supseteq(g \mu)^{G^{+}} \cap G \mu=S \mu,
$$

and so $\operatorname{cl}_{G}\left(g^{G}\right) \supseteq S \supseteq g^{G}$.

It is necessary to study conjugacy separability of the base group of $G^{+}$first. The next section is devoted to the study of $A^{+}=\pi_{1}\left(\left.\mathscr{G}\right|_{Y}, Y\right)$, where $Y$ is a maximal subtree of $X$. 


\section{CoNJUGaCY SEPARABILITY OF $A^{+}$}

We begin with the following general fact.

Proposition 4.1. Let $\mathscr{G}$ be a graph of groups over $X$, and assume that

$$
\bigcap_{P \in I} H_{e} P_{i(e)}=H_{e} \quad \text { for all } e \in E X,
$$

with $G=\pi_{1}(\mathscr{G}, X)$. Let $Y$ be a connected subgraph of $X$, and put $G(Y)=$ $\left\langle A_{x}: x \in V Y\right\rangle \leq G$. Then $G(Y)=\bigcap_{P \in I} G(Y) \operatorname{ker} \pi_{P}$. If $Y$ is finite then $G(Y)=\bigcap_{M \triangleleft_{f} G} G(Y) M$.

Proof. Fix a vertex $x_{0} \in V Y$, and for each edge $e \in E X$ let $T_{e} \ni 1$ be a left transversal of $H_{e}$ in $A_{i(e)}$. Then every element of $G$ is uniquely represented by a normal word $t_{1} \cdots t_{n} a$, where $a \in A_{x_{0}}, t_{i} \in T_{e_{i}}$ for $1 \leq i \leq n,\left(e_{1}, \ldots, e_{n}\right)$ is a closed path at $x_{0}$, and if $e_{i}=\bar{e}_{i-1}$ then $t_{i} \neq 1$ [8, Corollary 1]. Moreover, the proof shows that the elements of $G(Y)$ are represented by paths that entirely belong to $Y$. Now condition (1) means that, given any element $g=t_{1} \cdots t_{n} a$ in normal form in $G$, we can find $P \in I$ such that $\left(t_{1} \pi_{P}\right) \cdots\left(t_{n} \pi_{P}\right)\left(a \pi_{P}\right)$ is the normal form of $g \pi_{P}$ in $G_{P}$ (relative to suitable transversals of the $H_{e} \pi_{P}$ in the $\left.A_{i(e)} \pi_{P}\right)$. In particular, if $g \in G \backslash G(Y)$ then $g$ is represented by a path that goes outside $Y$, and since $g \pi_{P}$ is represented by the same path, we have $g \pi_{P} \notin G_{P}(Y)=\left\langle A_{x} \pi_{P}: x \in V Y\right\rangle=G(Y) \pi_{P}$. The first part follows.

Now suppose that $Y$ is finite. Then for any $P \in I$ we have the finitely generated subgroup $G(Y) \pi_{P}$ of the free-by-finite group $G_{P}$. Such subgroups are profinitely closed (e.g., [2, p. 229]). This evidently means that $G(Y) \operatorname{ker} \pi_{P}=$ $\bigcap_{M \in P \uparrow} G(Y) M$, and the second part follows (cf. the proof of 2.1).

To simplify the notation let $Y$ be a tree, $\mathscr{G}$ a graph of groups over $Y$, and $A=\pi_{1}(\mathscr{G}, Y)$. Also write $C=\bigcup_{x \in V Y} A_{x}$ and $D=\bigcup_{e \in E Y} H_{e}$, the subsets of vertex elements and edge elements respectively in $A$.

Proposition 4.2. Let $g$ be an element of $A$. Then the following assertions are true:

(i) If $g \in D$, then there exists a subtree $Y_{g}$ of $Y$ such that $g \in H_{e}$ if and only if $e$ is an edge of $Y_{g}$.

(ii) If $g \notin D$, then there exists a FINITE subtree $Y_{g}$ of $Y$ such that $g \in$ $A\left(Y_{g}\right)$, and if $Z$ is any subtree of $Y$ with $g \in A(Z)$, then $Y_{g} \subseteq Z$.

Proof. (i) Let $Y_{g}$ consist of all vertices $x$ of $Y$ such that $g \in A_{x}$, and all geodesics between them. If $g \in A_{x} \cap A_{y}$, and $p$ is the $Y$-geodesic from $x$ to $y$, then $g$ belongs to every vertex group of $p$ (since adjacent vertex groups in $A$ generate their amalgamated free product). In other words, $g$ belongs to every vertex group of $Y_{g}$. Moreover, $H_{e}=A_{i(e)} \cap A_{t(e)}$ for any edge $e$, so $g \in H_{e}$ if and only if $i(e)$ and $t(e)$ belong to $Y_{g}$.

(ii) Among all subtrees $Z$ with $g \in A(Z)$ pick one with the fewest number of vertices, and call it $Y_{g}$. Fix a vertex $x_{0}$ in $Y_{g}$. Then the normal form $t_{1} \cdots t_{n} a$ of $g$ can be represented by a closed path $p$ at $x_{0}$, with $p$ entirely contained in $Y_{g}$ (since $g \in A\left(Y_{g}\right)$ ). Now let $Z$ be a subtree such that $g \in A(Z)$. Suppose first that $Y_{g}=\left\{x_{0}\right\}$. If $x_{0} \notin V Z$ then $g \in A_{x_{0}} \cap A(Z)$ must be an edge element (consider the geodesic from $x_{0}$ to $A(Z)$ ), a contradiction. So in this case $Y_{g} \subseteq Z$. 
There remains the case where $Y_{g}$ has more than one vertex. If $Z$ contains every end vertex of $Y_{g}$ then $Y_{g} \subseteq Z$, so suppose $Z$ does not contain some end vertex $x$ of $Y_{g}$, and let $e$ be the edge of $Y_{g}$ that ends at $x$. Now the path $p$ must visit $x$ at least once (since otherwise $g \in A\left(Y_{g} \backslash\{e, x\}\right)$ ), and if $t_{i} \in A_{x}$ then $t_{i} \neq 1$ (since $p$ looks like $\ldots, e, x, \bar{e}, \ldots$ at $x$, and we have a normal form). If $Z$ and $Y_{g}$ were disjoint then $g \in A(Z) \cap A\left(Y_{g}\right)$ would be an edge element, which is not the case. Thus $Z^{\prime}=Z \cup Y_{g} \backslash\{e, x\}$ is a subtree of $Y$ such that $g \in A\left(Z^{\prime}\right)$. If $x_{0}$ is on $Z^{\prime}$ then we have the immediate contradiction that $g$ can be represented in normal form by a path $q$ in $Z^{\prime}$, and $q \neq p$ since $x$ is on $p$ but not on $q$. So we must have $x_{0}=x$. But since $Z$ and $Y_{g}$ are not disjoint we can always choose $x_{0}$ to be a common vertex. This final contradiction shows that $Z \supseteq Y_{g}$, as required.

To define the notion of a reduced form for elements of $A$ we need a definition. Let $\Delta^{\prime}$ be a nonempty subtree of a finite tree $\Delta$. By a reduction process $R$ from $\Delta$ to $\Delta^{\prime}$ we mean a sequence $\Delta_{1}, \ldots, \Delta_{m}$ of subtrees of $\Delta$ such that $\Delta_{1}=\Delta, \Delta_{m}=\Delta^{\prime}$, and $\Delta_{i+1}$ is obtained from $\Delta_{i}$ by deleting an end vertex $x_{i}$ (for $i=1, \ldots, m-1$ ). We let $y_{i}$ be the vertex of $\Delta_{i}$ adjacent to $x_{i}$, so when $\Delta \subseteq Y$ we have

$$
A\left(\Delta_{i}\right)=\left\langle A\left(\Delta_{i+1}\right) * A_{x_{i}}: H_{y_{i} x_{i}}=H_{x_{i} y_{i}} \text { via } \theta_{y_{i} x_{i}}\right\rangle, \quad i=1, \ldots, m-1 .
$$

Let $\Delta \supseteq \Delta^{\prime}$ be finite subtrees of $Y$, and $R=\left\{\Delta_{1}, \ldots, \Delta_{m}\right\}$ a reduction process from $\Delta$ to $\Delta^{\prime}$. Say $g$ is reduced (resp. cyclically reduced) relative to $R$ if either $g \in A\left(\Delta^{\prime}\right)$ or $g \in A\left(\Delta_{i}\right) \backslash A\left(\Delta_{i+1}\right)$ for some $i \leq m-1$, and then $g$ is reduced (resp. cyclically reduced) in the amalgamated free product $A\left(\Delta_{i}\right)$ of $A\left(\Delta_{i+1}\right)$ and $A_{x_{i}}$. Every element of $A(\Delta)$ can be written in reduced form, and is conjugate, in $A(\Delta)$, to a cyclically reduced element of $A(\Delta)$. Note that an elementm may be cyclically reduced relative to one reduction process, but not another.

Lemma 4.3. Assume that (1) holds. Let $g$ be a nonvertex element of $A$ such that $g$ is cyclically reduced relative to some reduction process from $Y_{g}$ to a single vertex. Then $\operatorname{cl}_{A}\left(g^{A}\right)=\bigcup_{\alpha} g_{\alpha}^{A}$, where each $g_{\alpha} \in A\left(Y_{g}\right)$.

Proof. There exists $P_{0} \in I$ such that $Y_{g \pi_{P}}=Y_{g}$ for all $P \subseteq P_{0}$. To begin with, we can choose $P_{1}$ such that $g \pi_{P}$ is cyclically reduced in $A\left(Y_{g}\right) \pi_{P}$ relative to $R$, for all $P \subseteq P_{1}$. This is because (1) can be used to ensure that the finitely many vertex elements in the cyclically reduced form of $g$ are excluded from the finitely many edge subgroups encountered by $R$. In particular, if $P \subseteq P_{1}$ then $g \pi_{P}$ is not an edge element of $A \pi_{P}$. For such $P$ we have $Y_{g \pi_{P}} \subseteq Y_{g}$ (since $g \pi_{P} \in A\left(Y_{g}\right) \pi_{P}$ ), and if $Q \subseteq P$ then $Y_{g \pi_{Q}} \supseteq Y_{g \pi_{P}}$. Since $Y_{g}$ is finite, there exists a subtree $\Delta \subseteq Y_{g}$ and $P_{0} \subseteq P_{1}$ such that for all $P \subseteq P_{0}$ we have $Y_{g \pi_{P}}=\Delta$. Then $g \in \bigcap_{P \subseteq P_{0}} A(\Delta) \operatorname{ker} \pi_{P}=A(\Delta)$ by 4.1 , and then $\Delta=Y_{g}$ by definition of $Y_{g}$.

Now suppose $P \subseteq P_{0}$. Then $g \pi_{P}$ is a cyclically reduced nonvertex element of $A\left(Y_{g}\right) \pi_{P}$, so the conjugacy theorem for amalgamated free products [9] implies that the conjugacy class of $g \pi_{P}$ in $A\left(Y_{g}\right) \pi_{P}$ contains no vertex elements. If $\Delta \supseteq Y_{g}$ is a finite subtree then the conjugacy class of $g \pi_{P}$ in $A(\Delta) \pi_{P}$ contains no vertex elements (consider a reduction process from $\Delta$ to $Y_{g}$ ). Now 
let $g^{\prime} \in \operatorname{cl}_{A}\left(g^{A}\right)$, and let $\Delta \supseteq Y_{g}$ be a finite subtree with $g^{\prime} \in A(\Delta)$. Let $R=\left\{\Delta_{1}, \ldots, \Delta_{m}\right\}$ be a reduction process from $\Delta$ to $Y_{g}$. Replacing $g^{\prime}$ by a conjugate in $A(\Delta)$ we may assume that $g^{\prime}$ is cyclically reduced relative to $R$, and we may choose $P_{0}$ such that $g^{\prime} \pi_{P}$ is cyclically reduced for all $P \subseteq P_{0}$. If $P \subseteq P_{0}$ then $g \pi_{P}$ has minimal length in its $A(\Delta) \pi_{P}$-conjugacy class, and belongs to $A\left(\Delta_{2}\right) \pi_{P} \backslash H_{y_{1} x_{1}} \pi_{P}$. The conjugacy theorem for amalgamated free products [9] implies that $g^{\prime} \pi_{P} \in A\left(\Delta_{2}\right) \pi_{P}$. Continuing in this way we finally obtain $g^{\prime} \pi_{P} \in A\left(Y_{g}\right) \pi_{P}$, whence $g^{\prime} \in \bigcap_{P \subseteq P_{0}} A\left(Y_{g}\right) \operatorname{ker} \pi_{P}=A\left(Y_{g}\right)$, as required.

We can now prove

Theorem 4.4. Let $g$ be a nonvertex element of $A$ such that $g$ is cyclically reduced relative to some reduction process $R$ from $Y_{g}$ to a vertex. Assume that (1) holds and that $H_{x_{1} y_{1}}$ is compact in the I-topology (where $x_{1} y_{1}$ is the first edge deleted in the reduction process). Then $g$ is conjugacy distinguished in $A$.

Proof. By 4.3 we have $\mathrm{cl}_{A}\left(g^{A}\right)=\bigcup g_{\alpha}^{A}$, where each $g_{\alpha} \in A\left(Y_{g}\right)$, and we may assume that each $g_{\alpha}$ is cyclically reduced relative to $R$. Fix $\alpha$, and choose $P_{0}$ such that for all $P \subseteq P_{0}, g \pi_{P}$, and $g_{\alpha} \pi_{P}$ are cyclically reduced in $A\left(Y_{g}\right) \pi_{P}$ relative to $R$. The conjugacy theorem for amalgamated free products can now be applied to deduce that $g_{\alpha} \pi_{P}$ is conjugate to some cyclic permutation of $g \pi_{P}$, via an element of $H_{x_{1} y_{1}} \pi_{P}$. Replacing $g$ by a cyclic permutation if necessary we have $g_{\alpha} \pi_{P}=\left(h_{P}^{-1} g h_{P}\right) \pi_{P}$ for all $P \subseteq P_{0}$, where $h_{P} \in H_{x_{1} y_{1}}$. Consider the function $f: H_{x_{1} y_{1}} \rightarrow A\left(Y_{g}\right)$ given by $f(h)=g_{\alpha}^{-1} h^{-1} g h$. With the appropriate profinitely topologies it is easy to see that $f$ is continuous and $\operatorname{ker} \pi_{P} \cap A\left(Y_{g}\right)$ is closed in $A\left(Y_{g}\right)$. Thus for each $P \subseteq P_{0}, f^{-1}\left(\operatorname{ker} \pi_{P}\right)$ is a nonempty closed subset of $H_{x_{1} y_{1}}$, and $P \subseteq Q$ implies that $f^{-1}\left(\operatorname{ker} \pi_{P}\right) \subseteq f^{-1}\left(\operatorname{ker} \pi_{Q}\right)$, so we have the finite intersection property. If

$$
h \in \bigcap_{P \subseteq P_{0}} f^{-1}\left(\operatorname{ker} \pi_{P}\right)=f^{-1}\left(\bigcap_{P \subseteq P_{0}} \operatorname{ker} \pi_{P}\right)=f^{-1}(\langle 1\rangle),
$$

then $g_{\alpha}^{-1} h^{-1} g h=1$, so $g_{\alpha} \in g^{A}$. The result follows.

The next lemma collects the information we need on conjugacy of edge and vertex elements.

Lemma 4.5. Let $C=\bigcup_{x \in V Y} A_{x}$ and $D=\bigcup_{e \in E Y} H_{e}$.

(i) Let $h \in D$, and let $a \in C$ be such that $h^{a} \in C$. Then $a \in A_{x}$ for some $x \in V Y_{h}$.

(ii) Let $a \in A_{x}, b \in A_{y}$, and $a^{b} \in C$. Then there exists a vertex $z$ such that $a, b \in A_{z}$.

(iii) Let $h \in D$, and $w$ be a nonvertex element of $A$ such that $h^{w} \in C$. Let $w=a_{1} \cdots a_{r}$ be the reduced form of $w$ (relative to some reduction process $R$ of $\left.Y_{w}\right)$, where the $a_{i} \in C$. Then $h^{a_{1} \cdots a_{i}} \in D$ for $1 \leq i \leq r-1$. $a^{A_{x}}$.

(iv) Let $a \in A_{x}$ be such that $a^{A_{x}} \cap D=\varnothing$. Then $a^{A} \cap D=\varnothing$, and $a^{A} \cap C=$

Proof. (i) Choose $x$ as close to $Y_{h}$ as possible subject to $a \in A_{x}$, and get a contradiction to $h^{a} \in C$ by assuming that $x \notin V Y_{h}$ and considering the geodesic from $x$ to $Y_{h}$. 
(ii) Similar to (i): choose $x_{1}$ and $y_{1}$ as close to each other as possible (on the geodesic joining $x$ and $y$ ) subject to $a \in A_{x_{1}}$ and $b \in A_{y_{1}}$.

(iii) First note that $Y_{h} \cap Y_{w} \neq \varnothing$, for otherwise a consideration of the geodesic from $Y_{h}$ to $Y_{w}$ gives the contradiction $h^{w} \notin C$. Thus $a=h^{w} \in A\left(Y_{w}\right)$. The proof is by induction on $r$, where $w=a_{1} \cdots a_{r}$. Consider the first step in the reduction process: if $h^{a_{1}}$ is not an edge element then $h^{w}$ is reduced as written (in the amalgamated free product of $A\left(\Delta_{2}\right)$ and $\left.A_{x_{1}}\right)$ and so cannot be a vertex element. Therefore $h_{1}=h^{a_{1}} \in D$, and $a=h_{1}^{a_{2} \cdots a_{r}}$. The result follows by induction on $r$ (using part (i)).

(iv) Suppose $a^{w}=h \in D$ for some nonvertex element $w=a_{1} \cdots a_{r}$, in reduced form. Then $h^{a_{r}^{-1} \cdots a_{1}^{-1}}=a \in C$, and by part (iii) we get $a^{a_{1}}=h^{a_{r}^{-1} \cdots a_{2}^{-1}} \in$ $D$. So $a^{A} \cap D \neq \varnothing$ implies that $a^{b} \in D$ for some vertex element $b$. By part (ii), $a$ and $b$ must belong to the same vertex group, which has to be $A_{x}$ since $a$ belongs to no other vertex group. But now we have $a^{A_{x}} \cap D \neq \varnothing$, a contradiction. It follows that $a^{A} \cap D=\varnothing$.

For the second part let $b=a^{w} \in A_{y}$, and choose $y$ as close to $x$ as possible subject to $b \in A_{y}$. Claim that $y=x$. For if not, then $Y_{w} \neq\{x\}$, and we have two cases to consider:

Case 1. $x \notin V Y_{w}$. Then there exists a finite subtree $\Delta \supseteq Y_{w}$ and an edge $y x$ with $y \in V \Delta$. Now $a \in A_{x} \backslash H_{x y}, w \in A(\Delta)$, and $b=w^{-1} a w \in A(\Delta)$ (as $\Delta$ has to contain $y$ because $b=a^{w} \in A(\Delta \cup\{x\})$ and $\left.y \neq x\right)$. This forces $w \in H_{y x}=H_{x y}$, and $a^{w} \in A(\Delta) \cap A_{x}=H_{x y}$, contradicting $a^{A} \cap D=\varnothing$.

Case 2. $x \in V Y_{w}$. Let $R=\left\{\Delta_{1}, \ldots, \Delta_{m}\right\}$ be a reduction process from $Y_{w}$ to a point, such that the first vertex deleted is $x_{1} \neq x$ (this can be done since $Y w \neq\{x\})$. Write $w=a_{1} \cdots a_{r}$ in reduced form relative to $R$. Then $b=$ $a_{r}^{-1} \cdots a_{1}^{-1} a a_{1} \cdots a_{r} \in A_{y} \subseteq A\left(\Delta_{2}\right) \cup A\left(x_{1}\right)$, so we must have $a_{1} \in A_{x}$ and $a^{a_{1}} \in H_{y_{1} x_{1}}$ (since $r \geq 2$ ). This is a contradiction.

We have now shown that $b=a^{w} \in A_{x}$. An argument similar to that in Case 2 now shows that $w \in A_{x}$, so $a^{A} \cap C \subseteq a^{A_{x}}$, are required.

We can now prove

Theorem 4.6. Let $g \in A_{x}$ be such that $g^{A_{x}} \cap D=\varnothing$. Assume that $X$ is locally finite, that every edge subgroup of $A$ is compact in the I-topology, and that $\bigcap_{P \in I} g^{A_{x}} P_{x}=g^{A_{x}}$. Then $g$ is conjugacy distinguished in $A$.

Proof. If $\mathrm{cl}_{A}\left(g^{A}\right)$ contains a nonvertex cyclically reduced element $g_{0}$, then by 4.4 we have $\mathrm{cl}_{A}\left(g^{A}\right)=\mathrm{cl}_{A}\left(g_{0}^{A}\right)=g_{0}^{A}$, so $g^{A}=g_{0}^{A}=\mathrm{cl}_{A}\left(g^{A}\right)$ and we are done. (Note that condition (1) is a consequence of the compactness assumption, so 4.4 is applicable.) We may therefore suppose that $\operatorname{cl}_{A}\left(g^{A}\right)=\bigcup a_{i}^{A}$, where each $a_{i}$ is a vertex element of $A$. Let $S=\bigcup_{i(e)=x} H_{e}$, and note that $S$ is compact since $X$ is locally finite. Put $T_{P}=g^{A_{x}} \pi_{P} \cap S \pi_{p}$. We claim that there exists $P_{0}$ such that $T_{P}=\varnothing$ for all $P \subseteq P_{0}$. Suppose not. Note that $Q \subseteq P$ implies that $T_{Q}$ maps into $T_{P}$ under the obvious map (from $A_{Q}$ to $A_{P}$ ), so if $T_{P} \neq \varnothing$ then we have an inverse system of nonempty finite sets. Let $\left(h_{P} \pi_{P}\right) \in \underset{\lim }{\leftarrow} T_{P}$, where each $h_{P} \in S$. Since $S$ is compact there exists $h \in S$ such that $\overleftarrow{h}_{P}=h_{P} \pi_{P}$ for all $P$. This clearly means that $h \in \bigcap_{P \in I} g^{A_{x}} P_{x}=g^{A_{x}}$, contradicting $g^{A_{x}} \cap D=\varnothing$. Hence there exists $P_{0}$ such that $g^{A_{x}} \pi_{P} \cap D \pi_{P}=\varnothing$ for all $P \subset P_{0}$. By 4.5(iv) 
we have $g^{A} \pi_{P} \cap D \pi_{P}=\varnothing$, and $a_{i} \pi_{P} \in g^{A} \pi_{P} \cap C \pi_{P}=g^{A_{x}} \pi_{P}$. Thus first of all $a_{i} \in \bigcap_{P \subseteq P_{0}} A_{x} \operatorname{ker} \pi_{P}=A_{x}$, and then $a_{i} \in \bigcap_{P \subseteq P_{0}} g^{A_{x}} P_{x}=g^{A_{x}}$. Thus $\operatorname{cl}_{A}\left(g^{A}\right)=g^{A}$, as required.

Remark 1. For $g$ as in 4.6, the condition $\bigcap_{P} g^{A_{x}} P_{x}=g^{A_{x}}$ is implied by $\mathrm{cl}_{A}\left(g^{A}\right)=g^{A}$. For if $b \in \bigcap g^{A_{x}} P_{x}$, then $b \in \mathrm{cl}_{A}\left(g^{A}\right)=g^{A}$, and so $b \in$ $g^{A} \cap C=g^{A_{x}}$ by $4.5($ iv) .

Remark 2. The usual proof of the conjugacy separability of the profinite group $\widehat{A_{x}}$ in fact shows that for $g \in \widehat{A_{x}}$ we have $\bigcap_{P \in I} g^{\widehat{A_{x}}} \bar{P}_{x}=g^{\widehat{A_{x}}}$.

The next two results give partial information about conjugacy separability of edge elements.

Lemma 4.7. Assume that (1) holds. Let $h$ be an edge element of $A$, and assume that there exists $P_{0} \in I$ such that $Y_{h \pi_{P_{0}}}=Y_{h}$. Then $\mathrm{cl}_{A}\left(h^{A}\right) \cap D=$ $\mathrm{cl}_{A\left(Y_{h}\right)}\left(h^{A\left(Y_{h}\right)}\right) \cap D$.

Proof. The condition implies that $h \pi_{P} \notin H_{e} \pi_{P}$ for all $P \subseteq P_{0}$, where $e$ is an edge leading out of $Y_{h}$. Let $z \in \mathrm{cl}_{A}\left(h^{A}\right) \cap D$, so $z \pi_{P}=h^{w} \pi_{P}$ for some $w \in A$. We claim that $w$ can be chosen in $A\left(Y_{h}\right)$. For if not, let $\Delta$ be the smallest tree containing $h \pi_{P}$ and $w \pi_{P}$, and consider a reduction process $\left\{\Delta_{1}, \ldots, \Delta_{m}\right\}$ from $\Delta$ to $\Delta \cap Y_{h}$. Now the first vertex $x_{1} \notin V Y_{h}$, and $w \pi_{P} \notin A\left(\Delta_{2}\right) \pi_{P}$ by the minimal choice of $\Delta$, and yet $\left(h \pi_{P}\right)^{w \pi_{P}}$ belongs to a vertex group. By the conjugacy theorem for amalgamated free product, we must have $h \pi_{P} \in H_{y_{1} x_{1}} \pi_{P}$, contradicting the choice of $P$. We have therefore shown that

$$
h_{0} \in \bigcap_{P \subseteq P_{0}} h^{A\left(Y_{h}\right)} \operatorname{ker} \pi_{P} \subseteq \bigcap_{P \subseteq P_{0}} A\left(Y_{h}\right) \operatorname{ker} \pi_{P}=A\left(Y_{h}\right)
$$

and so

$$
\begin{aligned}
h_{0} & \in \bigcap_{P \subseteq P_{0}} h^{A\left(Y_{h}\right)} \operatorname{ker} \pi_{P} \cap A\left(Y_{h}\right) \\
& =\bigcap_{P \subseteq P_{0}} h^{A\left(Y_{h}\right)}\left(\operatorname{ker} \pi_{P} \cap A\left(Y_{h}\right)\right)=\operatorname{cl}_{A\left(Y_{h}\right)}\left(h^{A\left(Y_{h}\right)}\right) .
\end{aligned}
$$

Let $h$ be an edge element of $A$. For any sequence $\sigma=\left(x_{1}, \ldots, x_{r}\right)$ of vertices of $A$ put $D_{\sigma}(h)=D \cap\left\{h^{a_{1} \cdots a_{r}}: a_{i} \in A_{x_{i}}\right\}$. We have

Theorem 4.8. Assume that $X$ is locally finite and $D$ is compact. Let $h$ be an edge element of $A^{+}$. Then $h$ is conjugacy distinguished in $A^{+}$if and only if $h^{A^{+}} \cap D=D_{\sigma}(h)$ for some finite sequence $\sigma$ of vertices.

Proof. We know that $\operatorname{cl}_{A^{+}}\left(h^{A^{+}}\right)=\bigcup h_{\alpha}^{A^{+}}$with each $h_{\alpha} \in D$. Let $K=h^{A^{+}} \cap D=$ $\bigcup_{\sigma} D_{\sigma}(h)$, the union being over all finite sequences $\sigma$. If $z \in \mathrm{cl}_{A^{+}}\left(h^{A^{+}}\right) \cap D$ and $P \in I$ then there exists a sequence $\sigma(P)$ such that $z \pi_{P} \in D_{\sigma(P)}(h) \pi_{P} \subseteq K \pi_{P}$, so $z \in K \operatorname{ker} \pi_{P}$. Thus

$$
\mathrm{cl}_{A^{+}}\left(h^{A^{+}}\right) \cap D \subseteq \bigcap_{P} K\left(\operatorname{ker} \pi_{P}\right)=\operatorname{cl}_{A^{+}}(K) .
$$

Now $D$ is a compact subset of $A^{+}$, and so is closed. Therefore $\mathrm{cl}_{A^{+}}(K)=$ $\operatorname{cl}_{D}(K)$. Conversely if $z \in \operatorname{cl}_{D}(K)$ and $P \in I$ then $z \pi_{P} \in K \pi_{P}$, so $z \pi_{P} \in$ 
$D_{\sigma}(h) \pi_{P}$ for some sequence $\sigma$ (depending on $P$ ). In other words, $z \in \mathrm{cl}_{A^{+}}\left(h^{A^{+}}\right)$, and we have shown that $\mathrm{cl}_{A^{+}}\left(h^{A^{+}}\right) \cap D=\operatorname{cl}_{D}\left(h^{A^{+}} \cap D\right)$. We also note that for a fixed sequence $\sigma$, the set $D_{\sigma}(h)$ is a complete subspace of the compact set $D$ (since each vertex group $\widehat{A}_{x}$ is compact), and is therefore compact. So if $h^{A^{+}} \cap D=D_{\sigma}(h)$ then $\mathrm{cl}_{A^{+}}\left(h^{A^{+}}\right) \cap D=\operatorname{cl}_{D}(K)=K=h^{A^{+}} \cap D$, which implies that every $h_{\alpha} \in h^{A^{+}}$, and so $h$ is conjugacy distinguished in $A^{+}$. Conversely, suppose $\mathrm{cl}_{A^{+}}\left(h^{A^{+}}\right)=h^{A^{+}}$. Then $K=h^{A^{+}} \cap D=\operatorname{cl}_{D}(K)$, so the compact (since closed) set $K$ is the (countable) union of the closed sets $D_{\sigma}(h)$. Enumerate the finite sequences $\left\{\sigma_{1}, \sigma_{2}, \ldots\right\}$, and put $T_{i}=\bigcup_{1 \leq j \leq i} D_{\sigma_{j}}(h)$. Then $K=\bigcup_{i=1}^{\infty} T_{i}$, and each $T_{i}$ is closed. By the Baire Category Theorem (or rather its proof, using compactness), some $T_{i_{0}}$ has nonempty interior, and so contains a set of the form $h P_{x} \cap D$, where $h \in D$ and $P \in I$. Since $P_{x} \triangleleft_{f} A_{x}$ and $K$ is compact, it is easy to see that $K$ is the union of finitely many $T_{i}$, whence $K=T_{j}$ for some $j$. Let $\sigma$ be the concatenation of the sequences $\sigma_{1}, \ldots, \sigma_{j}$. Then it is clear that $D_{\sigma_{i}}(h) \subseteq D_{\sigma}(h)$ for $1 \leq i \leq j$, and so $h^{A^{+}} \cap D=K=T_{j} \subseteq D_{\sigma}(h)$, as required.

The following are immediate consequences of 4.7 and 4.8.

Corollary 4.9. Let $X$ be locally finite, and let $h$ be an edge element of $A^{+}$such that $Y_{h}$ is finite. Then $h$ is conjugacy distinguished in $A^{+}$if and only if for some finite sequence $\sigma$ of vertices of $Y_{h}$ we have $h^{A^{+}} \cap D=D_{\sigma}(h)$.

Corollary 4.10. Let $A^{+}$be the amalgamated free product of countably many groups. Then an element $h$ of the associated subgroup $H$ is conjugacy distinguished in $A^{+}$if and only if $h^{A^{+}} \cap H=D_{\sigma}(h)$ for some finite sequence $\sigma$ of vertices.

\section{CONJUGACY SEPARABILITY OF $G^{+}$}

If $X$ is not a tree then $G^{+}$is a nontrivial $\mathrm{HNN}$-extension over the base group $A^{+}=\left\langle\hat{A}_{x}: x \in V X\right\rangle$. By the length $|g|$ of an element $g \in G^{+}$we mean the number of $t$-symbols appearing in an HNN-reduced form for $g$. Thus $g=a_{1} t_{e_{1}}^{\varepsilon_{1}} \cdots t_{e_{n}}^{\varepsilon_{n}} a_{n+1}$, where all $a_{i} \in A^{+}, a_{n+1}$ is arbitrary, each $\varepsilon_{i}= \pm 1$, each $e_{i} \in E X \backslash E Y$ (where $Y$ is a fixed maximal subtree of $X$ ), and if $e_{i-1}=e_{i}$ and $\varepsilon_{i-1}+\varepsilon_{i}=0$ then $a_{i} \notin H_{\varepsilon_{i} e_{i}}$ (we write $H_{-e}$ instead of $H_{\bar{e}}$ for ease of notation). The reduced element $g$ is cyclically reduced if $a_{n+1}=1$ and $t_{e_{n}}^{\varepsilon_{n}} a_{1} t_{e_{1}}^{\varepsilon_{1}} \cdots a_{n}$ is also reduced.

Theorem 5.1. Let $g \in G^{+}$be cyclically reduced and of length at least one. Then $g$ is conjugacy distinguished in $\mathrm{G}^{+}$.

Proof. Let $z$ be a cyclically reduced element in $\operatorname{cl}_{G^{+}}\left(g^{G^{+}}\right)$. It is easy to see that the edge subgroups $\bar{H}_{\varepsilon e}$ are closed in $A^{+}$, so we can find $P_{0} \in I$ such that $|g|=\left|g \pi_{P}\right|,|z|=\left|z \pi_{P}\right|$, and both $g \pi_{P}$ and $z \pi_{P}$ are cyclically reduced in $G_{P}$ for all $P \subseteq P_{0}$. The conjugacy theorem for HNN-extensions (e.g. [3]) now implies that $\left|g \pi_{P}\right|=\left|z \pi_{P}\right|$, so $|g|=|z|$. Modulo the same $P$ we know that $g \pi_{P}$ and some cyclic permutation of $z \pi_{p}$ have the same sequence of $t$ symbols with the same exponents. Replacing $z$ by a suitable cyclic permutation we may assume that $g=a_{1} t_{e_{1}}^{\varepsilon_{1}} \cdots a_{n} t_{e_{n}}^{\varepsilon_{n}}$ and $z=b_{1} t_{e_{1}}^{\varepsilon_{1}} \cdots b_{n} t_{e_{n}}^{\varepsilon_{n}}$. For $P \subseteq P_{0}$ the conjugacy theorem in $G_{P}$ implies the existence of elements $h_{0, P}, \ldots, H_{n, P}$ such that, modulo $P$ 


$$
\begin{aligned}
h_{0, P} b_{1} & \equiv a_{1} h_{1, P}, & & h_{1, P} \in \bar{H}_{\varepsilon_{1} e_{1}}, \\
\left(h_{1, P} \bar{\theta}_{e_{1}}^{\varepsilon_{1}}\right) b_{2} & \equiv a_{2} h_{2, P}, & & h_{2, P} \in \bar{H}_{\varepsilon_{2} e_{2}}, \\
\vdots & & & \\
\left(h_{n-1, P} \bar{\theta}_{e_{n-1} \varepsilon_{n-1}}\right) b_{n} & \equiv a_{n} h_{n, P}, & & h_{n, P} \in \bar{H}_{\varepsilon_{n} e_{n}}, \\
\left(h_{n, P} \bar{\theta}_{e_{n}}^{\varepsilon_{n}}\right) & \equiv h_{0, P}, & & \text { so } h_{0, P} \in \bar{H}_{-\varepsilon_{n} e_{n}}=\bar{H}_{\varepsilon_{0} e_{0}} .
\end{aligned}
$$

Since $\bigcup_{i=0}^{n} \bar{H}_{\varepsilon_{i} e_{i}}$ is compact, there exist elements $h_{i} \in \bar{H}_{\varepsilon_{i} e_{i}}$ such that $h_{i} \pi_{P}=$ $h_{i, P} \pi_{P}$ for all $P$. Then we have the equations $h_{0} b_{1}=a_{1} h_{1},\left(h_{1} \bar{\theta}_{e_{1}}^{\varepsilon_{1}}\right) b_{2}=$ $a_{2} h_{2}, \ldots, h_{n} \bar{\theta}_{e_{n}}^{\varepsilon_{n}}=h_{0}$, and so $z=h_{0}^{-1} g h_{0}$. The result follows.

To deal with conjugacy separability of elements of $A^{+}$in $G^{+}$, we begin with the following trivial observation, which can be proved by using a length argument (as in the proof of 5.1) and the conjugacy theorem for HNN-extensions.

Lemma 5.2. If $a \in A^{+}$then $\operatorname{cl}_{G^{+}}\left(a^{G^{+}}\right)=\bigcup a_{\alpha}^{G^{+}}$, where each $a_{\alpha} \in A^{+}$. In particular, $a$ is conjugacy distinguished in $G^{+}$if and only if $\mathrm{cl}_{G^{+}}\left(a^{G^{+}}\right) \cap A^{+}=$ $a^{G^{+}} \cap A^{+}$.

The conjugacy theorem for elements of the base of an HNN-extension states that if $a, b \in A^{+}$are conjugate in $G^{+}$, then there exist elements $h_{1}, \ldots, h_{m} \in$ $\bigcup_{e \in E X \backslash E Y} \bar{H}_{e}$, such that $a \sim h_{1}$ in $A^{+}$(i.e., conjugate in $A^{+}$), $b \sim h_{m}$ in $A^{+}$, and for $1 \leq i \leq m-1$, either $h_{i+1} \sim h_{i}$ in $A^{+}$or $h_{i+1}=h_{i} \theta_{e}$ for some $e \in E X \backslash E Y$. Now we have

Proposition 5.3. Let $a \in A^{+}$be a nonvertex cyclically reduced element (relative to some reduction process of $\left.Y_{a}\right)$. Then $\mathrm{cl}_{G^{+}}\left(a^{G^{+}}\right) \cap A^{+}=a^{A^{+}}$, and so $a$ is conjugacy distinguished in $\mathrm{G}^{+}$.

Proof. Choose $P_{0}$ such that for all $P \subseteq P_{0}$ we have $Y_{a \pi_{P}}=Y_{a}$ and $a \pi_{P}$ is cyclically reduced (cf. the proof of 4.4). Then $\left(a \pi_{P}\right)^{A^{+} \pi_{P}} \cap D \pi_{P}=\varnothing$ by 4.5(iii) and using the fact that no nonvertex conjugate of an edge element can be cyclically reduced. Now let $b \in \operatorname{cl}_{G^{+}}\left(a^{G^{+}}\right) \cap A^{+}$. Then $b \pi_{P}$ is conjugate to $a \pi_{P}$ in $G_{P}$. Since no $A^{+} \pi_{P}$-conjugate of $a \pi_{P}$ can belong to an edge subgroup in $G_{P}$, we must have $b \pi_{P}$ conjugate to $a \pi_{P}$ in $A^{+} \pi_{P}$, and so $b \in \mathrm{cl}_{A^{+}}\left(a^{A^{+}}\right)=$ $a^{A^{+}}$, the latter by 4.4. The final part follows from 5.2.

For vertex elements of $A^{+}$we have the following (recall that $C=\bigcup_{x \in V X} \widehat{A_{x}}$ ):

Proposition 5.4. Assume that $X$ is locally finite. Let a be a vertex element of $A^{+}$, and let $b \in \operatorname{cl}_{G^{+}}\left(a^{G^{+}}\right) \cap C$. Then there exists a sequence $c_{0}, c_{1}, \ldots$ of elements of $C$ such that (i) $c_{0}=a$; (ii) for each $i \geq 1$, either $c_{i} \sim c_{i-1}$ in $C$, or $c_{i}=c_{i-1} \theta_{e_{i}}$ for some $e_{i} \in E X \backslash E Y$; and (iii) $c_{i} \rightarrow b$ as $i \rightarrow \infty$.

Proof. For $P \in I$ let $S_{P}(a, b)$ denote the set of all sequences $\sigma=\left\{a_{i, P} \pi_{P}: i \geq\right.$ $0\}$, where each $a_{i, P} \in C, a_{0, P} \pi_{P}=a \pi_{P}, a_{i, P} \pi_{P}=b \pi_{P}$ for all sufficiently large $i$, and each $a_{i, P} \pi_{P}$ is either $C \pi_{P}$-conjugate to, or the $\theta_{e, P}$-image of, $a_{i-1, P} \pi_{P}$ (in other words, each $\sigma \in S_{P}(a, b)$ represents a possible conjugacy $a \pi_{P} \sim b \pi_{P}$ in $\left.G_{P}^{+}\right)$. For fixed $i$ and $P$ write $T_{i}(P)$ for the set of $i$ th components of all $\sigma \in S_{P}(a, b)$. Suppose $a \in \widehat{A}_{x}$, and let $\Delta_{i}$ denote the (finite) 
subtree of radius $i$ with centre $x$. A simple inductive argument shows that $T_{i}(P) \subseteq \bigcup_{y \in V \Delta_{i}} \hat{A}_{y} \pi_{P}$, so each $T_{i}(P)$ is finite. If $Q \subseteq P$ then every sequence in $S_{Q}(a, b)$ maps to a sequence in $S_{P}(a, b)$ under the induced map $G_{Q}^{+} \rightarrow G_{P}^{+}$, and so $T_{i}(Q)$ maps into $T_{i}(P)$.

We construct the elements $c_{i}$ inductively, beginning with $c_{0}=a$. Assume that $c_{1}, \ldots, c_{i-1}$ have been chosen subject to (ii) and $c_{j} \pi_{p} \in T_{j}(P)$ for all $P$, and $1 \leq j \leq i-1$. Let $K_{P}=\left\{c^{\prime} \pi_{P} \in T_{i}(P)\right.$ : there exists $\sigma \in S_{P}(a, b)$ such that $\sigma_{i-1}=c_{i-1} \pi_{P}$ and $\left.\sigma_{i}=c^{\prime} \pi_{P}\right\}$. Then each $K_{P} \neq \varnothing$, and if $Q \subseteq P$ then $K_{Q}$ maps into $K_{P}$ under the induced map $G_{Q} \rightarrow G_{P}$. Let $\left(d_{P} \pi_{P}\right)$ belong to the inverse limit of the sets $K_{P}$. Then each $d_{P} \in \bigcup_{y \in V \Delta_{i}} \widehat{A_{y}}$, and the latter set is compact, so there exists $c_{i} \in \bigcup_{y \in V \Delta_{i}} \widehat{A_{y}}$ such that $c_{i} \pi_{P}=d_{P} \pi_{P}$ for all $P$. Therefore, for every $P$ there exists $\sigma \in S_{P}(a, b)$ such that $\sigma_{i-1}=c_{i-1} \pi_{P}$ and $\sigma_{i}=c_{i} \pi_{P}$. Also $c_{i} \pi_{P} \in T_{i}(P)$ for all $P$. To verify (ii), note that for each $P$ the element $c_{i} \pi_{P}$ is either conjugate to $c_{i-1} \pi_{P}$ by some element of $\bigcup_{y \in V \Delta_{i-1}} \widehat{A_{y}} \pi_{P}$, or is the image of $c_{i-1} \pi_{P}$ under some $\theta_{e, P}$, where $e \in E \Delta_{i}$. The number of possibilities is therefore finite and independent of $P$, and so at least one must hold for all $P$. If $c_{i} \pi_{p}=c_{i-1} \pi_{P} \theta_{e, P}$ for all $P$, then $c_{i-1} \in \bar{H}_{e}$ and we get $c_{i} \pi_{P}=\left(c_{i-1} \theta_{e}\right) \pi_{P}$ for all $P$, whence $c_{i}=c_{i-1} \theta_{e}$. If $c_{i} \pi_{P} \sim c_{i-1} \pi_{P}$ in $\bigcup_{y \in V \Delta_{i}} \widehat{A_{y}} \pi_{P}$ for all $P$, then $c_{i} \sim c_{i-1}$ in $\bigcup_{y \in V \Delta_{i}} \widehat{A}_{y}$, using compactness. This proves (ii) for $c_{i}$, and so the inductive construction goes through. Finally given $P$ it is clear that $c_{i} \pi_{P}=b \pi_{P}$ for all sufficiently large $i$, so $c_{i} \rightarrow b$ as $i \rightarrow \infty$.

Corollary 5.5. Let $a \in A^{+}$be a vertex element. Then a is conjugacy distinguished in $G^{+}$if and only if $a^{G^{+}} \cap C$ is a closed subset of $A^{+}$.

Proof. By 5.2 and the proof of 5.3 we see that $\mathrm{cl}_{G^{+}}\left(a^{G^{+}}\right)=\bigcup a_{\alpha}^{G^{+}}$, where each $a_{\alpha} \in C$. By 5.4, for each $a_{\alpha}$ there exists a sequence $c_{i} \in C$ such that $c_{i} \sim a$ in $G^{+}$and $c_{i} \rightarrow a_{\alpha}$ as $i \rightarrow \infty$. Thus each $a_{\alpha} \in \mathrm{cl}_{A^{+}}\left(a^{G^{+}} \cap C\right)$, and so $\operatorname{cl}_{G^{+}}\left(a^{G^{+}}\right) \cap C \subseteq \mathrm{cl}_{A^{+}}\left(a^{G^{+}} \cap C\right)$. The reverse inclusion being trivial, we have $\operatorname{cl}_{G^{+}}\left(a^{G^{+}}\right) \cap C=\mathrm{cl}_{A^{+}}\left(a^{G^{+}} \cap C\right)$. The result follows.

Corollary 5.6. Let a be a vertex element of $A^{+}$, and let $D^{\prime}=\bigcup_{e \in E X \backslash E Y} \bar{H}_{e}$. If $a^{A^{+}} \cap D^{\prime}=\varnothing$, then a is conjugacy distinguished in $G^{+}$if and only if $a$ is conjugacy distinguished in $A^{+}$.

Proof. Since no $A^{+}$-conjugate of $a$ belongs to an associated subgroup, we have $a^{G^{+}} \cap A^{+}=a^{A^{+}}$by the conjugacy theorem for $\mathrm{HNN}$-extensions, and so $a^{G^{+}} \cap C=$ $a^{A^{+}} \cap C$. The result follows from 5.5 and the fact that $\operatorname{cl}_{A^{+}}\left(a^{A^{+}}\right)=\bigcup a_{\alpha}^{A^{+}}$, with all $a_{\alpha} \in C$ (this is a consequence of 4.4).

To deal with elements of $D^{\prime}=\bigcup_{e \in E X \backslash E Y} \bar{H}_{e}$ we need the following:

Lemma 5.7. If $D$ is compact, then so is $C=\bigcup_{x \in V X} \widehat{A_{x}}$.

Proof. It is sufficient to prove that $C$ is complete, since we can always regard $C$ as a subspace of the profinite completion $\widehat{A}^{+}$. So let $\left\{a_{P}: P \in I\right\}$ be a subset of $C$ such that for all $Q \subseteq P$ we have $a_{Q} \pi_{P}=a_{P} \pi_{P}$. If every $a_{P} \in D$, then the compactness of $D$ implies the existence of $h \in D$ such that $a_{P} \pi_{P}=h \pi_{P}$ for all $P$, and we are done. Now suppose that $a_{P_{0}} \pi_{P_{0}} \notin D \pi_{P_{0}}$ for some $P_{0}$. Then $a_{P} \pi_{P} \notin D \pi_{P}$ for all $P \subseteq P_{0}$, for if $a_{p} \in \widehat{A_{y}} \neq \widehat{A}_{x}$ for some $P \subseteq P_{0}$, 
then $a_{P_{0}} \pi_{P_{0}}=a_{P} \pi_{P_{0}} \in \widehat{A_{y}} \pi_{P_{0}} \cap \widehat{A_{x}} \pi_{P_{0}} \subseteq D \pi_{P_{0}}$, contrary to assumption. But now the compactness of $\widehat{A}_{x}$ gives the result.

Consider the set of all finite sequences $\sigma=\left(x_{1}, e_{1}^{\varepsilon_{1}}, \ldots, e_{n}^{\varepsilon_{n}}, x_{n+1}\right)$, where $x_{i} \in V X, e_{i} \in E X \backslash E Y$, and $\varepsilon_{i}= \pm 1$. For such a sequence let

$$
B_{\sigma}(h)=C \cap\left\{h^{g}: g=a_{1} t_{e_{1}}^{\varepsilon_{1}} \cdots t_{e_{n}}^{\varepsilon_{n}} a_{n+1}, a_{i} \in \widehat{A}_{x_{i}} \text { for all } i\right\} .
$$

Theorem 5.8. Assume that $X$ is locally finite and $D$ is compact. Then $h \in$ $D^{\prime}$ is conjugacy distinguished in $G^{+}$if and only if there exist finite sequences $\sigma_{1} \ldots, \sigma_{m}$ such that $h^{G^{+}} \cap C=\bigcup_{i=1}^{m} B_{\sigma_{i}}(h)$.

Proof. By 5.5 the element $h$ is conjugacy distinguished in $G^{+}$if and only if $K=h^{G^{+}} \cap C$ is closed in $A^{+}$. Since $C$ is compact (by 5.7) and therefore closed, this is equivalent to $K$ being closed in $C$, i.e., to $K$ being compact. Now $K=\bigcup_{\sigma} B_{\sigma}(h)$, the union being over the countably many sequences $\sigma$. An application of Baire's Category Theorem (cf. the proof of 4.8) shows that $K$ is compact if and only if it is the union of finitely many $B_{\sigma_{i}}(h)$. (One needs to show that each $B_{\sigma}(h)$ is closed. For this, use the compactness of the $\widehat{A}_{x}$ to show that $B_{\sigma}(h)$ is complete.)

\section{REFERENCES}

1. R. B. J. T. Allenby and C. Y. Tang, Conjugacy separability of certain 1-relator groups with torsion, J. Algebra 103 (1986), 619-637.

2. D. E. Cohen, Combinatorial group theory: A topological approach, Cambridge Univ. Press, 1989.

3. D. J. Collins, Recursively enumerable degrees and the conjugacy problem, Acta Math. 122 (1969), 115-160.

4. W. Dicks and M. J. Dunwoody, Groups acting on graphs, Cambridge Univ. Press, 1989.

5. J. L. Dyer, Separating conjugates in free-by-finite groups, J. London Math. Soc. (2) 20 (1979), 215-221.

6. _ - Separating conjugates in amalgamated free products and HNN extensions, J. Austral. Math. Soc. Ser. A 29 (1980), 35-51.

7. D. Gildenhuys and L. Ribes, Profinite groups and Boolean graphs, J. Pure Appl. Algebra 12 (1978), 21-47.

8. P. J. Higgins, The fundamental groupoid of a graph of groups, J. London Math. Soc. (2) 13 (1976), 145-149.

9. W. Magnus, A. Karrass, and D. Solitar, Combinatorial group theory, Wiley-Interscience, 1966.

10. V. N. Remeslennikov, Conjugacy in polycyclic groups, Algebra and Logic 8 (1969), 404-411.

11. $\ldots$, Groups that are residually finite with respect to conjugacy, Siberian Math. J. 12 (1971), 783-792.

12. L. Ribes, Introduction to pro-finite groups and Galois cohomology, Queen's Papers in Pure and Appl. Math., vol. 24, Queen's University, Kingston, Ontario, 1970.

13. J.-P. Serre, Cohomologie Galoisienne, Springer, Berlin, 1964.

14. _ _ Trees, Springer, Berlin, 1980.

15. M. Shirvani, On residually finite HNN-extensions, Arch. Math. 44 (1985), 110-115.

16. __, On residually finite graph products, J. Pure Appl. Algebra 49 (1987), 281-282.

17. __ A converse to a residual finiteness theorem of G. Baumslag, Proc. Amer. Math. Soc. 104 (1988), 703-706.

18. P. F. Stebe, Conjugacy separability of certain free products with amalgamation, Trans. Amer. Math. Soc. 156 (1971), 119-129. 
19. _ Conjugacy separability of certain Fuchsian groups, Trans. Amer. Math. Soc. 163 (1972), 173-188.

20. P. A. Zalesskii and O. V. Mel'nikov, Subgroups of profinite groups acting on trees, Math. USSR-Sb. 63 (1989), 405-424.

Department of Mathematics, University of Alberta, Edmonton, Alberta, T6G 2G1 Canada 